Anne Oommen-Halbach

\title{
Verankerung der Kinderrechte im Grundgesetz? Über die Geschichte und aktuelle Konjunktur einer Debatte
}

Als Ärztin für Kinder- und Jugendmedizin am Institut für Geschichte, Theorie und Ethik der Medizin der Heinrich-Heine-Universität Düsseldorf interessiere ich mich für Fragen des Kinderschutzes und der Kinderrechte aus medizinhistorischer, -theoretischer und -ethischer Perspektive. Während der COVID-19-Pandemie wurden die Freiheitsgrade von Kindern und Jugendlichen zu ihrem eigenen Schutz und dem Schutz Anderer erheblich eingeengt. Die vor diesem Hintergrund verstärkt geführte Diskussion um die Rechte der Kinder und deren mögliche Verankerung im deutschen Grundgesetz soll im vorliegenden Beitrag in einen historischen Zusammenhang eingebettet und verständlich gemacht werden.

\section{1 „Neue“ Sorgen um die Rechte der Kinder?}

Im Mai 2020 warnte die Kinderkommission des Deutschen Bundestages davor, die Bedürfnisse und Rechte von Kindern nicht aus den Augen zu verlieren (Kommission zur Wahrnehmung der Belange der Kinder 2020). Hintergrund dieser Mahnung waren die seit Mitte März 2020 im Zuge der Corona-Pandemie erstmalig eingeführten Maßnahmen zum Infektionsschutz, die durch vorübergehende Schließungen von Bildungs-, Betreuungs- und Sporteinrichtungen den Alltag und die Lebenswelten von Kindern veränderten. Auch die Monitoring-Stelle UN-Kinderrechtskonvention des Deutschen Instituts für Menschenrechte formulierte eine Stellungnahme zu Kinderrechte[n] in Zeiten der Corona-Pandemie, in der sie im Hinblick auf Kinder und Jugendliche auf den Zielkonflikt zwischen dem Schutz der Gesundheit einerseits und der Wahrung persönlicher Freiheiten andererseits hinwies. Den Schutzrechten der Kinder sei das gleichzeitig bestehende „Recht auf soziale Kontakte, soziale Teilhabe, Spiel sowie frühkindliche und schulische Bildung“ (Feige und Gerbig 2020, 3) gegenüber zu setzen, das in der UN-Kinderrechtskonvention festgeschrieben sei. Die Deutsche Akademie für Kinder und Jugendmedizin e. V. wies am 20. April 2020 darauf hin, dass „Kinder und Jugendliche [...] in den Entscheidungsprozessen [zu den Infektionsschutzmaßnahmen] nicht als Personen mit ebenbürtigen Rechten gesehen“ worden seien (Deutsche Akademie für Kinder- und Jugendmedizin e. V. 2020). Schließlich berief sich das Bundesjugendkuratorium im Dezember 2020 in seinem Zwischenruf Kinder- und

2 Open Access. (c) 2022 Anne Oommen-Halbach (c) BY-NC-ND Dieses Werk ist lizenziert unter der Creative Commons Attribution-NonCommercial-NoDerivatives 4.0 Lizenz.

https://doi.org/10.1515/9783110760361-013 
Jugendrechte in der Krise stärken! (Bundesjugendkuratorium 2020) bereits auf erste Studienergebnisse, die zeigten, dass sich junge Menschen zu wenig in die Gestaltungsprozesse der Krisensituation einbezogen fühlten (Andresen et al. 2020). Hiermit werden nur einige wenige öffentliche Stellungnahmen zitiert, die angesichts der Maßnahmen zur Eindämmung der COVID-19-Pandemie auf die erforderliche Achtung der Kinderrechte im Allgemeinen hinwiesen.

$\mathrm{Zu}$ dieser generellen Diskussion gesellte sich in der Öffentlichkeit eine spezifische Debatte um die Verankerung von Kinderrechten im Grundgesetz (GG). Dieses bereits vor Beginn der Pandemie im März 2018 im Koalitionsvertrag der regierenden Parteien (Bundesregierung 2018, S. 21) ${ }^{1}$ festgeschriebene Vorhaben gewann angesichts der Pandemie neue, tagesaktuelle Argumente. So wies etwa die Deutsche Akademie für Kinder- und Jugendmedizin darauf hin, die „bisherigen politischen Entscheidungen in der Krise zeigen einmal mehr, warum Kinderrechte in das Grundgesetz gehörten“ (Deutsche Akademie für Kinder- und Jugendmedizin 2020, 1). Trotz dieser aktuellen Argumentationsanleihen handelt es sich nicht etwa um ein neues Anliegen, sondern um eine historisch entwickelte und in verschiedenen Nuancen bereits wiederholt geführte Auseinandersetzung.

Der folgende Beitrag möchte sowohl die Entwicklung und Diskussion um die Kinderrechte im Allgemeinen als auch die spezifische Frage der Verankerung von Kinderrechten im deutschen GG in einen historischen Kontext stellen. Hierzu sollen einige Entwicklungslinien und Protagonisten der Kinderrechtsbewegung insbesondere im 20. Jahrhundert im Überblick vor- und dargestellt werden.

\section{2 Über die „vornehmste Aufgabe“ des letzten Jahrhunderts}

„Freie Kinder zu schaffen, wird die vornehmste Aufgabe dieses Jahrhunderts sein“ (Rilke 2016, 73) - schrieb Rainer Maria Rilke im Jahr 1902 in seiner Rezension des im gleichen Jahr in deutscher Übersetzung erschienenen Buches Das Jahrhundert des Kindes (Key 1902) der schwedischen Pädagogin Ellen Key (1849-1926). Im Hinblick auf die Formulierung und Unterzeichnung wesentlicher internationaler Vereinbarungen, die in der Tat im 20. Jahrhundert geschaffen wurden, sollte Rilke recht behalten. Nichts desto weniger reichen die Anfänge der Kinderrechtsent-

1 Vgl. Bundesregierung (2018) 21: „Wir werden Kinderrechte im Grundgesetz ausdrücklich verankern. Kinder sind Grundrechtsträger, ihre Rechte haben für uns Verfassungsrang. Wir werden ein Kindergrundrecht schaffen.“ 
wicklung in der Geschichte wesentlich weiter zurück. Zugleich scheint das Ziel der freien Kinder auch im 21. Jahrhundert noch nicht erreicht zu sein.

Die heutige Auseinandersetzung über die Rechte der Kinder bewegt sich in einem Spannungsfeld zwischen Forderungen nach Schutz und Fürsorge einerseits und Partizipation und Autonomie des Kindes andererseits. Am Beginn dieser Geschichte stand jedoch vornehmlich der Gedanke, dass das Leben von Kindern als solches schützenswert sei. Es sollte dabei weder im Besitz der Eltern noch im Besitz anderer Personen stehen, wie bereits Martin Luther (1483-1546) aus einer religiösen und später John Locke (1632-1704) aus einer philosophischen Perspektive schlussfolgerten (Liebel 2017, 31; siehe auch Alaimo 2002). Aber auch Forderungen nach gesellschaftlicher Partizipation von Kindern sind älter als gemeinhin angenommen, wie die bereits 1796 veröffentlichte Schrift The Rights of Infants von Thomas Spence (1750-1814) belegt, die insbesondere eine gesellschaftliche Teilhabe von Kindern unter wirtschaftlichen Aspekten nahelegte (Spence 1796). Die Impulse für kindliche Partizipationsrechte sind vielfältig und beschränken sich nicht auf den westlichen Kulturkreis. Manfred Liebel weist in diesem Zusammenhang auf Traditionen und Ordnungen in manchen Regionen Afrikas, in andinen Kulturen Südamerikas oder auch den Maya-Kulturen Mittelamerikas hin, die den Kindern eine altersunabhängige verantwortungsvolle Gesellschaftsposition und -rolle zuweisen. Dabei ist die Wahl eines Kindes als Bürgermeister eines Dorfes, wie sie im Hochland Boliviens und Perus bis heute üblich ist, ein besonders sprechendes Beispiel (Liebel 2017, 32).

\subsection{Erste internationale Vereinbarungen}

$\mathrm{Zu}$ den frühen internationalen Erklärungen zählte die Genfer Deklaration der Rechte des Kindes, die 1924 von der Vollversammlung des Völkerbundes verabschiedet wurde. Sie ist recht kurz und besteht aus fünf Absätzen, die vor allem den Schutz des Kindes in wirtschaftlicher, physischer und psychischer Hinsicht fordern. Dabei geht der Artikel 2 konkret auf den Schutz des hungernden, kranken, zurückgebliebenen, verirrten, verwaisten und verlassenen Kindes ein. Man könnte die Deklaration auch als eine Aufzählung von Verpflichtungen der Erwachsenen gegenüber dem verletzten oder verletzlichen Kind bezeichnen. Kinder wurden als Objekt der Fürsorge der Erwachsenen und des Staates und nicht als Träger eigener Rechte verstanden. „Die Gesetzgeber von Genf haben Rechte und Pflichten verwechselt“ (Korczak 1999, 401) resümierte der jüdisch-polnische Kinderarzt, Pädagoge und Schriftsteller Janusz Korczak (1878/79-1942), dessen Ansprüchen an das Recht des Kindes auf Achtung - so der ins Deutsche über- 
setzte Titel seiner 1928 erstmals auf Polnisch erschienenen Schrift - die Genfer Deklaration nicht genügte.

Vordenkerin der Genfer Deklaration war die britische Pädagogin Eglantyne Jebb (1876-1928), die vor allem die infolge des ersten Weltkriegs notleidenden Kinder im Blick hatte. Sie gründete 1919 ein Komitee zum Kampf gegen die Hungersnot (Fight the Famine Council), aus dem noch im gleichen Jahr der Save the Children Fund hervorging, der internationale Hilfsprojekte für Kinder durchführte. Die Save the Children International Union schließlich einigte sich auf den Wortlaut der Deklaration, die in 37 Sprachen übersetzt wurde.

Nach der Auflösung des Völkerbundes 1948 verlor die Genfer Deklaration ihre völkerrechtliche Grundlage; sie wurde allerdings in die Kinderrechtsdeklaration der International Union for Child Welfare aufgenommen. Es schloss sich eine über zehnjährige Debatte darüber an, ob es einer gesonderten Kinderrechtserklärung bedürfe oder ob die Rechte der Kinder in der Allgemeinen Erklärung der Menschenrechte von 1948 ausreichend vertreten seien. 1959 wurde schließlich eine erweiterte Erklärung zum Schutz der Kinder durch die Generalversammlung der Vereinten Nationen (UNO) verabschiedet. Der Unterschied zur Genfer Deklaration bestand vor allem darin, dass die Kinder erstmalig als eigene Rechtsträger angesehen wurden, wenn sich die Inhalte auch weiterhin mehr auf den Schutz als auf ein Mitspracherecht der Kinder konzentrierten. Auch dieser Vertrag war, wie die vorhergehende Genfer Deklaration, in ihrer Rechtswirksamkeit noch nicht bindend (Wapler 2015, 80-81; Schmahl 2020, 56-57).

\subsection{Forderungen nach kindlicher Autonomie und Partizipation}

In den 1920er Jahren gab es aber ebenso schon Vordenker, die die Selbstbestimmung und gesellschaftliche Partizipation von Kindern forderten. Sie räumten dem Mitspracherecht eine höhere Priorität ein als dem Schutz der Kinder. Zu ihnen zählte Janusz Korczak, der nach kinderärztlicher Tätigkeit 1912 die pädagogische Leitung eines Warschauer Waisenhauses übernahm, wo er gemeinsam mit seiner Mitarbeiterin Stefa Wilczyńska (1886-1942) pädagogische Konzepte erarbeitete und praktizierte, die den Kindern im Zusammenleben weitreichende Selbstbestimmungsrechte einräumten. Hierzu zählte bspw. die Schaffung eines von Kindern geführten Parlaments sowie eines Kameradschaftsgerichts (vgl. Korczak 1999, 305).

Diesen im praktischen Erziehungskonzept gelebten Respekt vor der Würde des Kindes fasste Korczak auch in prägnante, für den damaligen wie heutigen Zeitgeist provokante Worte: In Anlehnung an die Magna Charta Libertatum, die 1215 dem englischen Adel grundlegende Freiheiten zusprach, formulierte Korczak 
eine Magna Charta Libertatis, in der er die Freiheitsrechte der Kinder auf drei wesentliche Aspekte kondensierte: „1. Das Recht des Kindes auf seinen Tod, 2. Das Recht des Kindes auf den heutigen Tag und 3. Das Recht des Kindes, das zu sein, wie es ist“ (Korczak 1999, 45). Insbesondere das erste dieser drei Rechte scheint den in der Genfer Deklaration formulierten Bemühungen um den Schutz des Kindes zu widersprechen (Kerber-Ganse 2009, 31) und ist erklärungsbedürftig: Korczak wandte sich hier gegen eine die kindliche Freiheit einschränkende elterliche und erzieherische Überfürsorge von Kindern. Gemeint ist das Recht des Kindes auf sein eigenes, vom Willen der Erwachsenen unabhängiges Leben, in dem es sich nach kindlichem Ermessen auch gefährden darf. Korczaks „menschenrechtlich begründete Haltung zum Kind“ (Kerber-Ganse 2009, 120) durchzieht sein vielfältiges pädagogisches Werk auch jenseits seiner explizit kinderrechtlichen Schrift (Korczak 1999).

Ähnlich revolutionär wie die Ansichten zu kindlicher Selbstbestimmung von Janusz Korczak waren die Forderungen der sog. Moskauer Deklaration der Rechte des Kindes, die im Februar 1918 verhandelt wurden (Liebel 2015). Im Kontext der Russischen Revolution wurden hier reformpädagogische Konzepte gefordert, die den Kindern eine mit den Erwachsenen nahezu gleichberechtigte Stellung in der Gesellschaft einräumten. Kinder sollten nicht nur ihre Meinung frei äußern können, sondern an allen sie betreffenden Entscheidungen beteiligt werden. Ihre Erziehung und auch ihre Erzieher selbst ${ }^{2}$ sollten sie frei wählen können, womit ihnen sogar das Recht eingeräumt wurde, sich im Zweifelsfall von ihren Eltern zu trennen. Sie sollten ihren wachsenden Fähigkeiten und Neigungen entsprechend ins Bildungs- und Arbeitsleben involviert werden und frühzeitig eine aktive Stellung in der Gesellschaft einnehmen. Die Deklaration, ein in 17 Abschnitten ausformulierter Text, entstand aus der Arbeit einer Vereinigung sozial und politisch engagierter Pädagoginnen und Pädagogen, die sich Freie Erziehung der Kinder nannte und maßgeblich durch den russischen Pädagogen Konstantin Nikoleavich Ventcel (1857-1947) geprägt wurde. Mit dem Ziel einer freieren und gerechteren Gesellschaft wurden hierbei den Kindern Rechte eingeräumt, die nicht nur über die damaligen, sondern auch über die heute gesellschaftlich anerkannten Vorstellungen von Kinderrechten in einigen Punkten weit hinausgehen. Zwar hat diese Deklaration zu keinem Zeitpunkt offizielle Anerkennung erlangt, doch lassen sich Spuren ihres Konzeptes in der russischen Pädagogik finden, bspw. in

2 Eine ähnliche, wenn auch anders konnotierte Forderung findet sich in dem bereits erwähnten Klassiker von Ellen Key (Key 1902, 9-46: „Das Recht des Kindes, seine Eltern zu wählen“). Key verband dabei pädagogische mit eugenischen Absichten: Eltern sollten durch eine gezielte Wahl ihres Partners ein möglichst optimales Erbgut ihrer Nachkommen anstreben (Kerber-Ganse 2016, 23-25). 
Form sogenannter Kinderclubs. In der Geschichte der Kinderrechte war die Moskauer Deklaration fast vergessen, wie sich bereits dem Untertitel des diesbezüglichen Beitrags von Manfred Liebel ,aus der verborgenen Geschichte der Kinderrechte“ entnehmen lässt (Liebel 2015; Liebel 2017, 37-39).

Noch weniger bekannt in der Geschichte der Kinderrechtsbewegung ist der koreanische Kinderpsychologe und Schriftsteller Bang Jung Whan ${ }^{3}$ (1899-1931), dessen 1923 formulierte Three Commitments for Children auch als Koreanische Deklaration der Kinderrechte bezeichnet wurden (Lee und Jung 2015, 262). Sie enthalten sowohl Aspekte des Schutzes als auch der Selbstbestimmung von Kindern. $\mathrm{Zu}$ den Forderungen Bang Jung Whans zählte die gesellschaftliche Anerkennung des Respekts und der Würde von Kindern als menschliche Wesen (1), ein Verbot von bezahlter und unbezahlter Kinderarbeit (2) und schließlich die Schaffung und Bereitstellung von Spiel- und Lern(Frei)räumen für Kinder in Familie und Gesellschaft (3) (Lee und Jung 2015, 264-265).

Zusammenfassend lassen sich in der Kinderrechtsbewegung in der ersten Hälfte des letzten Jahrhunderts zeitgleich sowohl Bestrebungen nach vermehrtem Schutz und Fürsorge als auch nach Autonomie und Partizipation von Kindern ausmachen. Während erstere durch die Genfer Deklaration internationale Anerkennung erfuhren, erhielten letztere zeitgenössisch nur wenig Aufmerksamkeit.

\section{Die Kinderrechtskonvention der Vereinten Nationen}

Die Kinderrechtskonvention (KRK), die vor über 30 Jahren am 20. November 1989 von der UN-Generalversammlung angenommen wurde und bereits zehn Monate später in Kraft trat, gilt als erste international völkerrechtlich bindende Übereinkunft, die die Selbstbestimmungsrechte von Kindern konkret benennt.

Einen ersten, an der Erklärung von 1959 orientierten Entwurf zu einer solchen Übereinkunft lieferte Polen im Jahr 1979. Jedoch erst dessen 1980 revidierte und wesentlich liberalere Fassung sollte zur Grundlage für die Formulierung der späteren Kinderrechtskonvention werden. An deren Erstellung arbeiteten in den folgenden 10 Jahren die 43 Mitglieder der UN-Menschenrechtskommission, einige UN-Sonderorganisationen sowie einige Nichtregierungsorganisationen (NGO) mit (Schmahl 2020, 57-58).

3 Es existieren in der Literatur unterschiedliche Schreibweisen des aus dem Koreanischen übertragenen Namens. 
Die komplexe Konvention aus insgesamt 54 Artikeln wird oft vereinfachend ${ }^{4}$ als Gebäude dargestellt, in denen das Kindeswohl (Artikel 3) als übergeordnetes Anliegen (Dach) von den Säulen aus Schutz-, Förderungs- und Beteiligungsrechten gehalten wird. Dabei soll das Kindeswohl5, verstanden als Gesamtwohl des Kindes hinsichtlich seines psychischen, emotionalen, sozialen, religiösen oder kulturellen Wohlergehens (Dörries, 2003), als Kriterium "bei allen Maßnahmen, die Kinder betreffen“ (BMFSFJ 2018, 12), berücksichtigt werden. Neben diesem Kriterium des Kindeswohls wurden drei weitere Artikel als übergeordnete Grundprinzipien bezeichnet, zu ihnen zählen das Recht auf Gehör und Berücksichtigung der kindlichen Meinung (Art. 12), das Recht auf Entwicklung (Art. 6) sowie das Recht auf Nicht-Diskriminierung (Art. 2) (Krappmann 2019, 315).

Die inhaltlich vielfältigen Artikel der Konvention tragen der Komplexität des Kindseins Rechnung, das in einem Spannungsfeld zwischen einer entwicklungsbedingt abnehmenden Abhängigkeit und Schutzbedürftigkeit und einer zugleich wachsenden Fähigkeit zu Autonomie und Mitsprache verortet werden kann (Wapler 2015, 84-86; Oommen-Halbach und Fangerau 2019). Die verschiedenen, in der Konvention festgeschriebenen Rechte, zu denen wirtschaftliche, soziale, kulturelle, bürgerliche und politische Rechte gleichermaßen zählen, sind daher auch nicht teilbar.

Im Vergleich zu früheren internationalen Vereinbarungen, die Kinderrechte betreffen, unterscheidet sich die KRK vor allem durch ihre Rechtsverbindlichkeit und die Aufnahme von Autonomie- bzw. Partizipationsrechten der Kinder. Diese finden insbesondere Ausdruck in Artikel 12 (Berücksichtigung des Kindeswillens), Artikel 13 (Meinungs- und Informationsfreiheit), Artikel 14 (Gedanken-, Gewissens- und Religionsfreiheit) sowie Artikel 15 (Vereinigungs- und Versammlungsfreiheit).

Die Vereinbarungen der KRK sind bindend für Deutschland (KRK, Art. 4), vergleichbar mit dem Rang eines einfachen Bundesgesetzes - damit steht die Kinderrechtskonvention nach den innerstaatlichen Regeln in Deutschland unter dem GG. Und genau daran entzündet(e) sich eine Debatte, die seit der Gültigkeit der Kinderrechtskonvention in Deutschland - hier wurde sie im Jahr 1992 ratifiziert $^{6}$ geführt wird.

4 Diese Darstellung wird der Komplexität der Konvention in einigen Aspekten nicht gerecht. Denn die Zuordnung der einzelnen Artikel und deren Absätze zu nur einer der drei Kategorien ist nicht immer zweifelsfrei möglich. Insbesondere Förderungs- und Beteiligungsrechte lassen sich nicht immer klar voneinander differenzieren.

5 Es wird in der internationalen Literatur und auch im englischen Wortlaut der Konvention als best interests of the child umschrieben.

6 Deutschland ratifizierte die KRK zunächst unter Vorbehalten, die erst 2010 zurückgenommen wurden. 


\section{Sollen Kinderrechte ins Grundgesetz und damit in die Verfassung aufgenommen werden?}

Diese Debatte suggeriert zunächst einmal die Annahme, dass Kinder bislang vom GG ausgeschlossen seien. Jedoch sind Kinder - wie alle anderen Menschen auch sehr wohl Grundrechtsträger. Sie werden allerdings als Rechtssubjekte mit ihrem - durch Entwicklung und Schutzbedürftigkeit bedingten - besonderen Status nicht explizit hervorgehoben (Artikel 6 und 7 erwähnen die Kinder zwar, aber nicht als Träger eigener Rechte, sondern vielmehr im Rahmen von Regelungen über Kinder im Kontext der Familie oder der Schule).

Der UN-Ausschuss für die Rechte des Kindes, ein Gremium, das die nationale Verwirklichung der Kinderrechtskonvention überprüft, empfahl bereits 2004 eine solche Verankerung zu überdenken. 10 Jahre später, im Jahr 2014, regte der gleiche Ausschuss an, der Kinderrechtskonvention im deutschen Recht einen höheren Rang zu verschaffen, ohne jedoch damit zwingend eine Grundgesetzänderung zu fordern (Wapler 2017, 9-10). Die National Coalition Deutschland, ein Netzwerk verschiedenster Nichtregierungsorganisationen und Initiativen zur Umsetzung der KRK, hingegen forderte eine explizite Grundgesetzänderung bereits mehrfach (National Coalition 2007; National Coalition 2019, 8-9).

Seit 1992 gab es vielfältige Vorschläge ${ }^{7}$ unterschiedlicher Interessensvertretungen für eine derartige Grundgesetzänderung. Konkrete Formulierungsvorschläge betrafen meist den Artikel 6 (Rechte der Eltern im Kontext der Familie), seltener den Artikel 2 (Recht auf Entfaltung der Persönlichkeit, Recht auf Leben und körperliche Unversehrtheit) des GG. Ohne deren Formulierungen im Einzelnen zu betrachten, können die wesentlichen Regelungsabsichten und -ziele der unterschiedlichen Akteure aus Politik, Recht und Kinderrechts-orientierter Interessensvertretung in Anlehnung an die Expertise der Verfassungsrechtlerin Friederike Wapler folgendermaßen als Thesen verkürzt werden (Wapler 2017, 17-19):

1. Die Verankerung von Kinderrechten im GG verdeutlicht eine symbolische Aufwertung der Subjektstellung des Kindes, wodurch das öffentliche Bewusstsein für die Belange der Kinder gestärkt wird.

2. Die Verankerung von Kinderrechten im GG kann den Besonderheiten der kindlichen Entwicklung Rechnung tragen.

7 Eine chronologische Auflistung der konkreten Vorschläge und ihrer Autoren lässt sich nachlesen bei Wapler (2017) 13-17. 
3. Die Verankerung von Kinderrechten im GG kann die Verantwortung des Staates für den Schutz und die Förderung kindgerechter Lebensbedingungen zum Ausdruck bringen.

4. Durch eine Verankerung von Kinderrechten im GG kann den Anliegen von Kindern im Kontext von behördlichen, gerichtlichen und politischen Entscheidungen im Sinne des Kindeswohlprinzips mehr Gewicht beigemessen werden (vgl. Artikel 3 Abs. 1 der KRK - Kindeswohlprinzip).

5. Die Verankerung von Kinderrechten im GG wird Kindern und Jugendlichen das Einklagen ihrer Rechte im Rahmen einer Verfassungsbeschwerde ermöglichen.

6. Die Verankerung von Kinderrechten im GG könnte die Aufwertung der Kindergrundrechte gegenüber dem Erziehungsrecht der Eltern bewirken.

In der Diskussion solcher Annahmen in Fachgremien aber auch in der breiten Öffentlichkeit erfolgte nicht selten eine Verquickung mit Anliegen und Gesetzesinitiativen des Kinderschutzes, z. B. mit dem am 1.1.2012 in Kraft getretenen Bundeskinderschutzgesetz.

Darüber hinaus wurden diese Debatten auch begleitet von Initiativen zur Festschreibung von Kinderrechten auf Landesebene. In den Landesverfassungen aller Bundesländer finden sich heute unterschiedliche Bestimmungen, die im weitesten Sinne die Kinderrechte betreffen (Monitoring-Stelle UN-Kinderrechtskonvention 2019).

\subsection{Entwurf der Bundesregierung zur Änderung des Grundgesetzes (2021)}

„Um aber die Sichtbarkeit von Kinderrechten und ihre Anwendung in der Praxis zu verbessern, sieht die Bundesregierung eine Änderung des GG als verfassungspolitisch sinnvolles und wichtiges Vorhaben an“ (BMFSFJ 2019, 4). Auf der Basis dieser bereits im Koalitionsvertrag der regierenden Parteien festgelegten Absicht ${ }^{8}$, verabschiedete das Bundeskabinett im Januar 2021 einen Gesetzentwurf, der eine Erweiterung des bestehenden Artikel 6, Absatz 2 vorsah.

Pflege und Erziehung der Kinder sind das natürliche Recht der Eltern und die zuvörderst ihnen obliegende Pflicht. Über ihre Betätigung wacht die staatliche Gemeinschaft. (Grundgesetz für die Bundesrepublik Deutschland, Art. 6, Abs. 2)

8 Vgl. Fußnote 1. 
Der bisherige Absatz beschreibt das Dreiecksverhältnis zwischen Kind, Eltern und Staat: Während die Eltern die Hauptverantwortung für ihre Kinder tragen, kommt der staatlichen Gemeinschaft die Aufgabe eines Wächters zu, der einspringt, wenn die Eltern ihren Pflichten nicht ausreichend nachkommen. Der jüngste Gesetzentwurf sah eine Ergänzung dieses Artikels um die folgenden Sätze vor:

Die verfassungsmäßigen Rechte der Kinder einschließlich ihres Rechts auf Entwicklung zu eigenverantwortlichen Persönlichkeiten sind zu achten und zu schützen. Das Wohl des Kindes ist angemessen zu berücksichtigen. Der verfassungsrechtliche Anspruch von Kindern auf rechtliches Gehör ist zu wahren, die Erstverantwortung der Eltern bleibt unberührt. (Deutscher Bundestag 2021, Gesetzentwurf der Bundesregierung)

In diesem vielfach kritisierten Gesetzentwurf der regierenden Parteien, der flankiert wurde durch alternative Vorschläge der Oppositionsparteien, werden unterschiedliche, bereits in der Vergangenheit formulierte Anliegen miteinander vereint (insbesondere die oben in Thesen formulierten Anliegen unter 1, 2, 4 und 5). Zugleich enthält dieser Entwurf drei der vier Grundprinzipien der KRK (Recht auf Gehör, Recht auf Entwicklung, Kindeswohlprinzip).

Das Echo des Gesetzentwurfs fiel geteilt aus: Insbesondere aus verfassungsrechtlicher Sicht wurde darauf hingewiesen, dass es im GG keine Schutzlücken im Hinblick auf die Grundrechte der Kinder gäbe, noch nicht einmal hinsichtlich der Einklagbarkeit der Grundrechte der Kinder vor dem Verfassungsgericht (z. B. Wapler 2021, 27-28). Es handele sich also lediglich um eine symbolische Aufwertung der Stellung des Kindes im GG, die zudem sprachlich missverständlich und unzureichend formuliert sei (Wapler 2021). ${ }^{9}$

Verschiedene Organisationen aus der Kinder- und Jugendhilfe, der Medizin und der (Sozial) Pädagogik hingegen kritisierten den Gesetzentwurf, weil er weit hinter der Kinderrechtskonvention zurückbliebe: Die Deutsche Akademie für Kinder- und Jugendmedizin wies auf das gänzliche Fehlen des Förderaspektes hin. Auch entspräche eine „angemessene“10 Beachtung des Kindeswohls nicht der in Artikel 3 der KRK formulierten „,vorrangigen“ Berücksichtigung (Deutsche Akademie für Kinder- und Jugendmedizin 2021). Das Aktionsbündnis Kinderrecht ${ }^{11}$

9 Noch schärfer in der Formulierung fiel die Ablehnung durch die Bundesrechtsanwaltskammer aus (vgl. Bundesrechtsanwaltskammer 2021).

10 In der Bundestagsdebatte am 15.04.2021 (erste Lesung) erläuterte Thorsten Frei die Formulierung „angemessen“ im Gesetzentwurf mit folgendem Beispiel: Dies könne bedeuten, dass bspw. ein Kinderspielplatz verkleinert werden müsse, damit ein Krankenhaus vergrößert werden könne.

11 Hierzu gehören das Deutsche Kinderhilfswerk, der Deutsche Kinderschutzbund, UNICEF Deutschland sowie die Deutsche Liga für das Kind. 
veranlasste einen Aufruf (Aktionsbündnis Kinderrecht 2021), der von einem breiten Bündnis verschiedenster Organisationen unterstützt wurde. Hier wurde insbesondere gefordert, die Kinderrechte in inhaltlich umfassender Form in einem eigenen Absatz im Grundgesetz zu verankern und nicht - wie bisher geplant diese in einem Absatz mit dem Elternrecht zu verknüpfen.

Ein in der Debatte wiederkehrendes Element stellte die Sorge dar, die Grundgesetzänderung könne zu einer Veränderung des Gefüges aus Kind, Eltern und Staat zugunsten des Staates führen. Eine solche Sorge fand nicht nur Niederschlag in der Bundestagsdebatte, sondern auch in den Stellungnahmen der evangelischen und katholischen Kirchen (Stellungnahme des Kommissariats der Deutschen Bischöfe 2021; Evangelische Arbeitsgemeinschaft Familie 2021).

In der im Frühsommer 2021 kontrovers geführten Bundestagsdebatte konnte schließlich keine notwendige Mehrheit der Fraktionen für den Gesetzentwurf erzielt werden. Damit ging erneut eine Gesetzesinitiative zur Verankerung von Kinderrechten im Grundgesetz ergebnislos zu Ende. Dennoch hat auch diese Debatte zu einer stärkeren öffentlichen Aufmerksamkeit für die Partizipationsrechte von Kindern geführt.

\section{Zusammenfassung}

Der Diskurs um die Rechte der Kinder blickt auf eine lange Geschichte zurück, an dessen Anfang vor allem die Schutzrechte der Kinder standen. In den 1920er Jahren entstanden zeitgleich mit der ersten internationalen Vereinbarung zum Kinderschutz auch Forderungen nach gesellschaftlicher Emanzipation und Partizipation von Kindern. Jedoch erst die KRK der Vereinten Nationen vereinte Schutz- und Partizipationsrechte im Rahmen einer völkerrechtlich bindenden Konvention. Seit der Ratifizierung der KRK in Deutschland 1992 wurde über die Verankerung von Kinderrechten im Grundgesetz nachgedacht, zuletzt während der 19. Legislaturperiode der Bundesrepublik Deutschland.

Dabei stand die Diskussion um die Rechte der Kinder im Frühsommer 2021 unter dem Eindruck der Pandemie und den Infektionsschutzmaßnahmen, die im besonderen Maße die Lebenswelten von Kindern einengten. Hierbei wurde die im Verlauf des Pandemiegeschehens gewonnene Erkenntnis, dass Kinder und Jugendliche hinsichtlich ihrer Bedürfnisse zu wenig gehört und in die politischen Entscheidungen zum Infektionsschutz einbezogen worden waren (Andresen et al. 2020), auch als Argument für die Notwendigkeit der Verankerung von Kinderrechten im Grundgesetz verwendet (Deutsche Akademie für Kinder- und Jugendmedizin e. V. 2020, 1): „Die politischen Diskussionen und getroffenen Maßnahmen in 
Deutschland haben gezeigt, dass Kinder als Träger:innen eigenständiger Rechte schnell übersehen werden“ - resümierte die Monitoring-Stelle UN-Kinderrechtskonvention in ihrer Stellungnahme im Mai 2020.

Dabei fehlte die Stimme von Kindern und Jugendlichen bislang weitgehend nicht nur im Diskurs über ihre spezifischen Problemlagen in der Pandemie, sondern auch in der allgemeinen Auseinandersetzung um die Umsetzung von Kinderrechten.

In diesem Sinne ist es zu begrüßen, dass vor Erstellung des Fünften und Sechsten Staatenberichts der Bundesrepublik Deutschland zu dem Übereinkommen der Vereinten Nationen über die Rechte der Kinder im Jahr 2019 immerhin erstmalig Kinder und Jugendliche $\mathrm{zu}$ ihrer Perspektive als Betroffene befragt wurden (BMFSFJ 2019, 3).

\section{Literatur}

Aktionsbündnis Kinderrecht (2021) Kinderrechte ins Grundgesetz - aber richtig!, https://www. dkhw.de/fileadmin/Redaktion/1_Unsere_Arbeit/1_Schwerpunkte/2_Kinderrechte/2.17_ Kinderrechte_ins_Grundgesetz/Appell_Kinderrechte_ins_Grundgesetz_-_aber_richtig_1705-21.pdf. Zugriff am 24. September 2021.

Alaimo K (2002) Historical roots of children's rights in Europe and the United States. In: Alaimo K, Klug B ( $\mathrm{Hg}$ ) Children as equals: Exploring the rights of the child. University Press of America, Lanham (Maryland), 1-23.

Andresen S, Lips A, Möller R, Rusack T, Schröer W, Thomas S, Wilmes J (2020) Erfahrungen und Perspektiven von jungen Menschen während der Corona-Maßnahmen. Erste Ergebnisse der bundesweiten Studie JuCo. Universitätsverlag Hildesheim, Hildesheim.

Bundesjugendkuratorium (2020) Zwischenruf des Bundesjugendkuratorium. Kinder- und Jugendrechte in der Krise stärken (15.12.2020). https://bundesjugendkuratorium.de/data/ pdf/press/bjk_zwischenruf_2020_jugendrechte_in_der_krise_stärken.pdf.Zugriff am 16. August 2021.

Bundesministerium für Familie, Senioren, Frauen und Jugend (BMFSFJ) (2018) Übereinkommen über die Rechte des Kindes. VN-Kinderrechtskonvention im Wortlaut mit Materialien. https://www.bmfsfj.de/resource/blob/93140/78b9572c1bffdda3345d8d393acbbfe8/ uebereinkommen-ueber-die-rechte-des-kindes-data.pdf.Zugriff am 17. September 2021.

Bundesministerium für Familie, Senioren, Frauen und Jugend (BMFSFJ) (2019) Fünfter und Sechster Staatenbericht der Bundesrepublik Deutschland zu dem Übereinkommen der Vereinten Nationen über die Rechte des Kindes. https://www.bmfsfj.de/resource/blob/ 141860/7c8b22d6eed03d0b378f0bfb39bbedc1/5-und-6-staatenbericht-der-brd-zumuebereinkommen-ueber-die-rechte-des-kindes-data.pdf. Zugriff am 20. September 2021.

Bundesrechtsanwaltskammer, Stellungnahme Nr. 16 (2021) Regierungsentwurf eines Gesetzes zur Änderung des Grundgesetzes zur ausdrücklichen Verankerung der Kinderrechte. https://brak.de/zur-rechtspolitik/stellungnahmen-pdf/stellungnahmen-deutschland/ 2021/februar/stellungnahme-der-brak-2021-16.pdf. Zugriff am 20. September 2021. 
Bundesregierung (2018) Ein neuer Aufbruch für Europa. Eine neue Dynamik für Deutschland. Ein neuer Zusammenhalt für unser Land. Koalitionsvertrag zwischen CDU, CSU und SPD, 19. Legislaturperiode, Berlin. https://archiv.cdu.de/system/tdf/media/dokumente/ koalitionsvertrag_2018.pdf?file=1. Zugriff am 16. August 2021.

Deutsche Akademie für Kinder- und Jugendmedizin e. V. (2020) Stellungnahme zu weiteren Einschränkungen der Lebensbedingungen von Kindern und Jugendlichen in der Pandemie mit dem neuen Coronavirus (SARS-CoV-2). https://www.dakj.de/wp-content/uploads/ 2020/04/2020-DAKJ-Stellungnahme-Lock-Down.pdf. Zugriff am 14. August 2021.

Deutsche Akademie für Kinder- und Jugendmedizin (DAKJ) Presseinformation (13.07.2021) Verankerung von Kinderrechten im Grundgesetz überfällig. https://www.dakj.de/ pressemitteilungen/dakj-presseinformation-verankerung-von-kinderrechten-imgrundgesetz-ueberfaellig/. Zugriff am 21. September 2021.

Deutscher Bundestag (2021) Gesetzentwurf der Bundesregierung. Entwurf eines Gesetzes zur Änderung des Grundgesetzes zur ausdrücklichen Verankerung der Kinderrechte vom 19.01.2021. https://dserver.bundestag.de/btd/19/281/1928138.pdf.Zugriff am 17. September 2021.

Dörries A (2003) Der Best-Interest-Standard in der Pädiatrie - theoretische Konzeption und klinische Anwendung. In: Wiesemann C, Dörries A, Wolfslast G, Simon A (Hg.) Das Kind als Patient. Ethische Konflikte zwischen Kindeswohl und Kindeswille. Campus, Frankfurt am Main und New York, 116-130.

Evangelische Arbeitsgemeinschaft Familie (2021) Kinderrechte und Grundgesetz - eaf-Alternativvorschlag zum Erreichen der Zweidrittelmehrheit. https://www.eaf-bund.de/sites/ default/files/2021-08/210119_PP_Kinderrechte_final.pdf.Zugriff am 15. September 2021.

Feige J, Gerbig S, Deutsches Institut für Menschenrechte, Monitoring-Stelle UN-Kinderrechtskonvention (2020) Stellungnahme Kinderrechte in Zeiten der Corona-Pandemie. Kinderrechtsbasierte Maßnahmen stützen und schützen. Kinder und Jugendliche in Krisenzeiten. https://www.institut-fuer-menschenrechte.de/fileadmin/Redaktion/Publikationen/ Stellungnahme_Kinderrechte_in_der_Corona-Pandemie.pdf. Zugriff am 12. August 2021.

Grundgesetz für die Bundesrepublik Deutschland Art 6. https://www.gesetze-im-internet.de/ gg/art_6.html. Zugriff am 21. September 2021.

Kerber-Ganse W (2016) Zur Geschichte der Kinderrechte. Zeitschrift für Museum und Bildung 80/81:21-32.

Kerber-Ganse W (2019) Die Menschenrechte des Kindes. Die UN-Kinderrechtskonvention und die Pädagogik von Janusz Korczak. Versuch einer Perspektivenverschränkung. Budrich, Opladen.

Key E (1902) Das Jahrhundert des Kindes: Studien. Fischer, Berlin.

Kommissariat der Deutschen Bischöfe (2021) Stellungnahme zum Entwurf eines Gesetzes zur Änderung des Grundgesetzes zur ausdrücklichen Verankerung der Kinderrechte BT-Drs.: 19/28138. https://www.kath-buero.de/files/Kath_theme/Stellungnahmen/ 2021/Stellungnahme_GE\%20Aenderung\%20des\%20Grundgesetzes\%20zur \%20ausdruecklichen\%20Verankerung\%20der\%20Kinderrechte_2021_05_17.docx.pdf. Zugriff am 15. September 2021.

Kommission zur Wahrnehmung der Belange der Kinder (Pressemitteilung) (2020) Bedürfnisse und Rechte von Kindern in der Pandemie nicht aus dem Blick verlieren. https://www. bundestag.de/resource/blob/694638/ac02d6edf056ef6bd6d111385fe1637d/Situationder-Kinder-in-der-Coronapandemie-data.pdf. Zugriff am 12. August 2021. 
Korczak J (1999) Das Recht des Kindes auf Achtung. In: Beiner F, Dauzenroth E (Hg) Sämtliche Werke Bd. 4. Gütersloher Verlagshaus, Gütersloh, 383-413.

Krappmann L (2019) Kinderrechte und Menschenrechte. In: Drerup J, Schweiger G (Hg) Handbuch Philosophie der Kindheit. J. B. Metzler, Stuttgart, 307-318.

Lee Y, Jung B (2015) Bang Jung Whan - The Korean Pioneer of Children's Rights. International Journal of Children's Rights 23:261-271.

Liebel M (2015) Die Moskauer Deklaration der Rechte des Kindes von 1918 - ein Beitrag aus der verborgenen Geschichte der Kinderrechte. Sozialwissenschaftliche Literatur-Rundschau. Zeitschrift für Sozialarbeit, Sozialpädagogik, Sozialpolitik und Gesellschaftspolitik 38:73-90.

Liebel M (2017) Kinderrechtsbewegungen und die Zukunft der Kinderrechte. In: Maier-Höfer C

$(\mathrm{Hg})$ Kinderrechte und Kinderpolitik. Fragestellungen der Angewandten Kindheitswissenschaften. Springer, Wiesbaden, 29-59.

Monitoring-Stelle UN-Kinderrechtskonvention (2019) Kinderrechte in den Landesverfassungen. https://landkarte-kinderrechte.de/kinderrechte-in-den-verfassungen-derbundeslaender/. Zugriff am 20. September 2021.

National Coalition (2007) Diskussion zur Aufnahme von Kinderrechten in die Verfassung. https://netzwerk-kinderrechte.de/wp-content/uploads/2021/06/2007_-Kinderrechte_in_ die_Verfassung.pdf. Zugriff am 17. September 2021.

National Coalition (2019) Die Umsetzung der UN-Kinderrechtskonvention in Deutschland, 5./6. Ergänzender Bericht an die Vereinten Nationen, 8-9. https://netzwerk-kinderrechte.de/ wp-content/uploads/2021/06/2007_-Kinderrechte_in_die_Verfassung.pdf.Zugriff am 17. September 2021.

Oommen-Halbach A, Fangerau H (2019) Selbstbestimmung von Kindern in der Medizin. In: Drerup J, Schweiger G (Hg) Handbuch Philosophie der Kindheit. J. B. Metzler, Stuttgart, 274-281.

Rilke RM (2016) Das Jahrhundert des Kindes. In: Guth KM (Hg) Rainer Maria Rilke. Aufsätze und Rezensionen. Hofenberg, Berlin, 72-76.

Schmahl S (2020) Kinderrechte im internationalen Recht in Geschichte und Gegenwart. In: Richter I, Krappmann L, Wapler F (Hg) Kinderrechte. Handbuch des deutschen und internationalen Kinder- und Jugendrechts. Nomos, Baden-Baden, 55-67.

Spence T (1796) The rights of infants, http://www.thomas-spence-society.co.uk/rights-ofinfants/. Zugriff am 18. August 2021.

Wapler F (2015) Kinderrechte und Kindeswohl. Mohr Siebeck, Tübingen.

Wapler F (2017) Kinderrechte ins Grundgesetz? In: Sachverständigenkommission 15. Kinderund Jugendbericht (Hg.) Materialien zum 15. Kinder und Jugendbericht. Zwischen Freiräumen, Familie, Ganztagsschule und virtuellen Welten - Persönlichkeitsentwicklung und Bildungsanspruch im Jugendalter. https://www.dji.de/fileadmin/user_upload/bibs2017/ 15_KJB_Wapler_b.pdf.Zugriff am 17. September 2021.

Wapler F (2021) Und ewig grüßt das Kindeswohl. „Kinderrechte ins Grundgesetz“: der Groundhog Day des Verfassungsrechts. https://verfassungsblog.de/und-ewig-grust-daskindeswohl. Zugriff am 16. September 2021. 